\title{
Simulation and Visualization of Air Flow Around Bat Wings During Flight
}

\author{
I.V. Pivkin ${ }^{1}$, E. Hueso ${ }^{2}$, R. Weinstein ${ }^{2}$, \\ D.H. Laidlaw ${ }^{2}$, S. Swartz ${ }^{3}$, and G.E. Karniadakis ${ }^{1}$ \\ ${ }^{1}$ Division of Applied Mathematics \\ ${ }^{2}$ Department of Computer Science \\ ${ }^{3}$ Department of Ecology and Evolutionary Biology, \\ Brown University, Providence, RI 02912, USA
}

\begin{abstract}
This paper presents a case study of interdisciplinary collaboration in building a set of tools to simulate and visualize airflow around bat wings during flight. A motion capture system is used to generate 3D coordinates of infrared markers attached to the wings of a bat flying in a wind tunnel. Marker positions that cannot be determined due to high wing deformation are reconstructed on the basis of the proper orthogonal decomposition (POD). The geometry obtained for the wings is used to generate a sequence of unstructured tetrahedral meshes. The incompressible Navier-Stokes equations in arbitrary LagrangianEulerian formulation are solved using the hybrid spectral/hp element solver Nektar. Preliminary simulation results are visualized in the CAVE, an immersive, 3D, stereo display environment.
\end{abstract}

\section{Introduction}

Fluid dynamics approaches have revolutionized our understanding of insect flight, revealing aerodynamic mechanisms almost unimaginable only 25 years ago [1-9]. Although bat and bird flight is also likely to yield insights of use in future technological application, such as the development of unmanned micro-air vehicles $[10,11]$, researchers of vertebrate flight are just beginning to incorporate in their work sophisticated methodologies drawn from the physical and mathematical sciences [12-14]. The unique features of bats - their specialized skeletal anatomy, high muscular control over wing conformation, and highly deformable wing-membrane skin - yield wings that undergo large changes in 3D geometry with every wing-beat cycle, and consequently produce highly maneuverable and energetically efficient flight [15-18].

To date, however, bat flight has not been studied from the quantitative perspective of unsteady aerodynamics. In particular, there is no rigorous understanding of the mechanisms by which the bat generates the high aerodynamic coefficients necessary for its flight capabilities, or of the vortex structure associated with the bat's exquisite aerodynamic control. Simulation of airflow around wings that not only flap but also undergo enormous shape changes in each wing-beat poses significant technical challenges, as does visualizing the complex 3D data such studies necessitate. 
This paper describes our interdisciplinary collaboration to build a set of tools for simulation and visualization of airflow around bat wings during flight. The next section gives a brief overview of our data acquisition techniques. In section 3 we discuss data preprocessing, which is necessary to provide input for the numerical simulations described in section 4 . Finally, the visualization of preliminary simulation results is presented in section 5 .

\section{Data Acquisition}

The motion-capture data of bat flight were acquired by flying more than 20 individuals of several species through a wind tunnel [18]. Two high-speed digital cameras tracked infrared markers attached to the bat wings. We chose to study the flight of a Pteropus Policephalus because of its large size and relatively slow motion.

After selecting 160 video frames where the motion of the bat is close to one complete wing beat, the Peak [19] motion capture system was used to extract the 3D coordinates of markers. These data are utilized to animate a simple 3D polygonal model of the surface of the bat's wings [20] (Fig. 1). Due to high wing deformations during flight, the $3 \mathrm{D}$ coordinates of a few markers cannot be determined in some frames. At the heart of generating polygonal bat wing model is the ability to reconstruct accurately the positions of these markers.
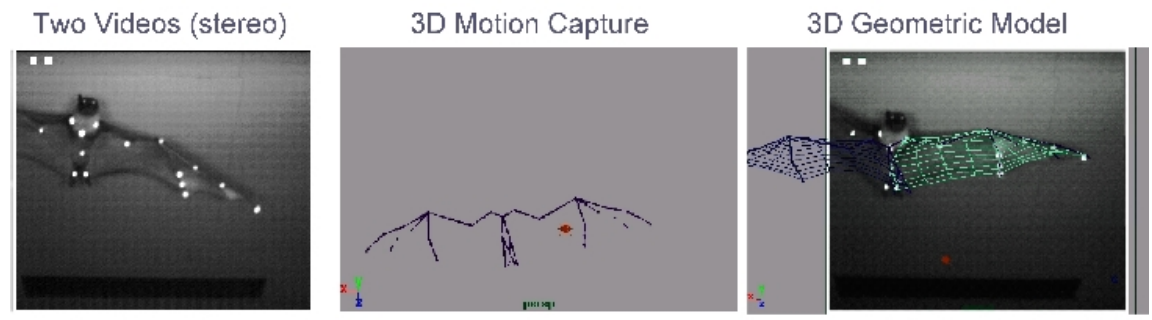

Fig. 1. Video capture of bat wing shape changes

\section{Missing Data Reconstruction}

Here we follow an approach based on proper orthogonal decomposition (POD) combined with the least-squares approach first proposed in [21] for image reconstruction. Let us consider a vector of data points $\mathbf{u}(t) \in\left(\Re^{3}\right)^{N}$ given for each frame $t$, where $N$ is the number of markers attached to the bat wings. We assume that we have available a finite number $P$ of frames. We then look for a representation of $\mathbf{u}(t)$ in the form

$$
\mathbf{u}(t)=\varphi_{0}+\sum_{k=1}^{N} \alpha_{k} \boldsymbol{\varphi}_{k},
$$


where $\boldsymbol{\varphi}_{0}=\sum_{t=1}^{P} \mathbf{u}(t)$ is a mean (time-averaged) position of the markers and $\boldsymbol{\varphi}_{k}$ are the orthonormal spatial modes. The unknown functions $\varphi_{k}$ can be calculated by minimizing an energy functional, producing a POD or Karhunen-Loeve decomposition.

The formulation assumes the completeness of the data; modifications are required if there exist a space-time regions in which components of $\mathbf{u}(t)$ are missing or corrupted. The procedure proposed in [21] completes the missing data iteratively starting from the average value at missing data location as the initial guess for the unknowns.

In order to make the bat motion cyclic, as is desirable for numerical simulations, we enforce periodicity on the POD mode coefficients $\alpha_{k}(t)$.

\section{Numerical Simulations}

The animated polygonal model is the basis for a sequence of tetrahedral meshes of the volume of 10 by 10 by 20 around the bat geometry, which has a wing span of approximately two non-dimensional units at its widest. Wings are represented by an infinitely thin tessellation of triangles (Fig. 2). An arbitrary Lagrangian-Eulerian (ALE) formulation of the incompressible Navier-Stokes equations is employed to solve for the flow field. This allow us to run simulations with changing geometry without remeshing each time step. A single tetrahedral mesh can be deformed to fit a number of frames, typically between 7 and 15 depending of the rate of deformation. When the deformation of the mesh becomes too extreme, elements degenerate and a new tetrahedral mesh must be created. As a result, multiple meshes are necessary and must be interpolated together in order to simulate an entire wing-beat. The mesh generator Gridgen [22] is used to generate up to 15 meshes for one wing-beat. Each mesh has approximately 6000 spectral tetrahedral elements. The governing equations are solved using the hybrid spectral/hp element solver Nektar [23].

Preliminary simulations were performed using third-order polynomial expansion in each element. The Reynolds number was set to 100 , greatly reducing computational

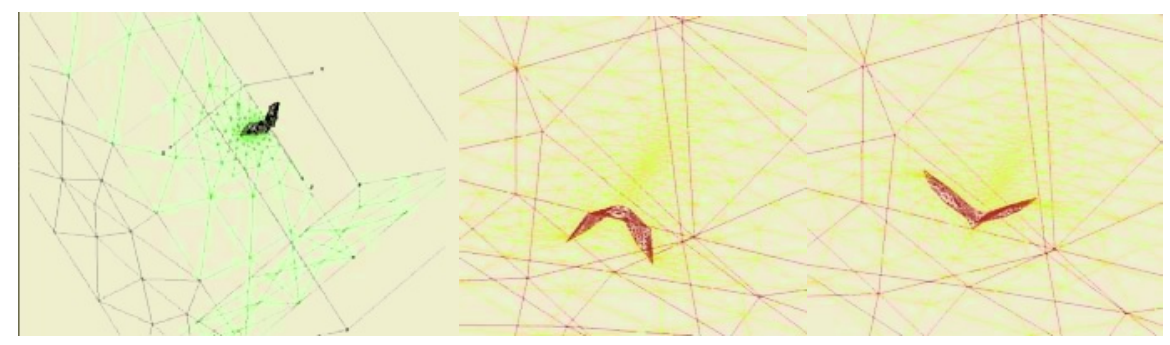

Fig. 2. Some of the meshes used in simulations 
effort and time. The solver produced 40 snapshots over one periodic motion cycle that describe the fluid velocity and the pressure distribution around the animated bat wings.

\section{Visualization}

The size and complexity of the time varying fields generated by the simulation makes them unfit for real-time visualization. To overcome this limitation, we pre-compute and store sets of pathlines and streamlines that can later be visualized interactively. The line sampling and visualization methods used on the bat flow data is a variation of the one presented in [24] for the visualization of blood flow in a coronary artery.

We visualize the flow data in the CAVE [25], an immersive 3D stereo display environment that scientists find more engaging than our less sophisticated desktop displays. One of our visualizations shows massless particles flowing down precomputed pathlines that resemble eels of variable length, color and opacity. A second visualization relies on animated streamlines, represented with lines of variable color and opacity. The user has interactive control over the number of lines displayed, how randomly they are distributed in space, and the mapping of opacity to flow quantities. These controls allow users to explore a continuum between localized visualization of detected vortices and the contextual flow around them [26] (Fig. 3).
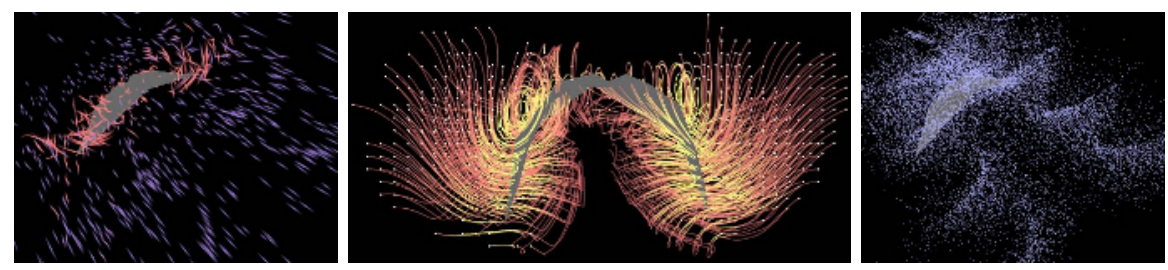

Fig. 3. Three different visualization methods are used to show different characteristics of the structure of the simulated flow around a motion-captured bat: (left to right) particle eels display pathlines, time-varying streamlines show vortices atop the wing during a down beat, and white dots capture structures in the wake

\section{Discussion and Conclusions}

Our studies differ from previous studies of bat flight in an important way: we do not treat the wings as simple oscillating plates, but instead explicitly characterize the changing intrinsic wing structure of potentially great aerodynamics importance. This makes realistic Reynolds number simulations challenging. The preliminary results reported here were obtained for $\mathrm{Re}=100$, too low to make judgments about the mechanisms by which the bat executes its flight capabilities. Even with higher Reynolds number simulations, the results would ideally be compared with 
experimental measurements to validate our process. Our current simulation results let us develop prototype visualization techniques that we believe can be extended to the flows with higher, more realistic Reynolds numbers. However, we have found that the preprocessing time and complexity of the visualization tools make them difficult to use on an everyday basis. Initial feedback does show that these tools help significantly in the exploration of complex time-dependent flow data.

In conclusion, we have completed a full iteration of simulation and visualization of unsteady flow around the bat wings during the flight. We believe that progress in understanding the relationship among wing shape, movement, and airflow during bat flight benefits significantly from the kind of interdisciplinary collaboration presented in this paper.

\section{Acknowledgements}

This work was supported in part by NSF (CCR-0086065 and CNS-0427374).

\section{References}

1. M.H. Dickinson and K.G. Gotz. Unsteady aerodynamic performance of model wings at low Reynolds numbers. Journal of Experimental Biology, 174:45-64, 1993.

2. M. H. Dickinson, F.O. Lehmann, and S.P. Sane. Wing rotation and the aerodynamic basis of insect flight. Science, 284(5422):1954-1960, 1999.

3. M.H. Dickinson. Unsteady mechanisms of force generation in aquatic and aerial locomotion. American Zoologist, 36(6):537-554, 1996.

4. R. Dudley. The biomechanics of insect flight: form, function, evolution. Princeton University Press, 2000.

5. C.P. Ellington. The aerodynamics of hovering insect flight. IV. Aerodynamic mechanisms. Phil. Trans. Roy. Soc. Lond. B, 305(1122):1-15, 1984.

6. C.P. Ellington. Limitations on animal flight performance. Journal of Experimental Biology, 160:71-91, 1991.

7. C. P. Ellington. Leading-edge vortices in insect flight. Nature, 384(6610):626-630, 1996.

8. S. Vogel. Flight in drosophila. II. Variations in stroke parameters and wing contour. $J$. Exp. Biol., 46:383-392, 1967.

9. A.P. Willmott, C.P. Ellington, and A.L.R. Thomas. Flow visualization and unsteady aerodynamics in the flight of the hawkmoth, manduca sexta. Philosophical Transactions of the Royal Society of London Series B - Biological Sciences, 352(1351):303-316, 1997.

10. C.P. Ellington. The novel aerodynamics of insect flight: Applications to micro-air vehicles. Journal of Experimental Biology, 202(23):3439-3448, 1999.

11. G.R. Spedding and P.B.S. Lissaman. Technical aspects of microscale flight systems. Journal of Avian Biology, 29(4):458-468, 1998.

12. J.M.V. Rayner, P.W. Viscardi, S. Ward, and J.R. Speakman. Aerodynamics and energetics of intermittent flight in birds. American Zoologist, 41:188-204, 2001.

13. G.R. Spedding, A. Hedenstrom, and M. Rosen. Quantitative studies of the wakes of freely flying birds in a low-turbulence wind tunnel. Experiments in Fluids, 34(2):291-303, 2003.

14. B.W. Tobalske, T.L. Hedrick, K.P. Dial, and A.A. Biewener. Comparative power curves in bird flight. Nature, 421:363-366, 2003. 
15. Y. Winter, C. Voigt, and O. Von Helversen. Gas exchange during hovering flight in a nectar-feeding bat glossophaga soricina. Journal of Experimental Biology, 201(2):237244, 1998.

16. C.C. Voigt and Y. Winter. Energetic cost of hovering flight in nectar-feeding bats (phyllostomidae : Glossophaginae) and its scaling in moths, birds and bats. Journal of Comparative Physiology B - Biochemical Systemic and Environmental Physiology, 169(1):38-48, 1999.

17. E.F. Stockwell. Morphology and flight manoeuvrability in new world leaf-nosed bats (chiroptera : Phyllostomidae). Journal of Zoology, 254:505-514, 2001.

18. Sharon Swartz, K. Bishop, and M.F. Ismael-Aguirre. Dynamic complexity of wing form in bats: implications for flight performance. In Functional and evolutionary ecology of bats. Oxford Press, 2005. In Press.

19. Peak performance, http://www.peakperform.com.

20. R. Weinstein, I. Pivkin, S. Swartz, D.H. Laidlaw, G. Karniadakis, and K. Breuer. Simulation and visualization of air flow around bat wings during flight. Technical Report CS-02-16, Brown University Computer Science Department, August 2002.

21. R.M. Everson and L. Sirovich. The Karhunen-Loeve transform of incomplete data. Journal of the Optical Society of America, A, 12(8):1657, 1995.

22. Gridgen, http://www.pointwise.com.

23. G.E. Karniadakis and S.J. Sherwin. Spectral/hp Element Methods for CFD. Oxford University Press, 1999.

24. J. Sobel, A. Forsberg, D.H. Laidlaw, R. Zeleznik, D. Keefe, I. Pivkin, G. Karniadakis, P. Richardson, and S. Swartz. Particle flurries: Synoptic 3D pulsatile flow visualization. IEEE Computer Graphics and Applications, 24(2):76-85, March/April 2004.

25. C. Cruz-Neira, D.J. Sandin, and T.A. DeFanti. Surround-screen projection-based virtual reality: The design and implementation of the CAVE. In Proceedings of ACM SIGGRAPH, volume 27, pages 135-142. ACM, August 1993.

26. E. Hueso. Visualizing vortices in simulated air flow around bat wings during flight. Technical Report CS-03-25, Master's Project, Computer Science Department, Brown University, Providence, RI, December 2003. 